\title{
Motion detection of a micromechanical resonator embedded in a d.c. SQUID
}

\author{
S. ETAKI ${ }^{1,2 *}$, M. POOT ${ }^{1}$, I. MAHBOOB ${ }^{2}$, K. ONOMITSU ${ }^{2}$, H. YAMAGUCH ${ }^{2}$ AND H. S. J. VAN DER ZANT ${ }^{1 *}$ \\ ${ }^{1}$ Kavli Institute of Nanoscience, Delft University of Technology, Post Office Box 5046, 2600 GA Delft, Netherlands \\ ${ }^{2} \mathrm{NTT}$ Basic Research Laboratories, NTT Corporation, Atsugi-shi, Kanagawa 243-0198, Japan \\ *e-mail: s.etaki@tudelft.nl; h.s.j.vanderzant@tudelft.nl
}

Superconducting quantum interference devices (SQUIDs) are the most sensitive detectors of magnetic flux ${ }^{1}$ and are also used as quantum two-level systems (qubits) ${ }^{2}$. Recent proposals have explored a novel class of devices that incorporate micromechanical resonators into SQUIDs to achieve controlled entanglement of the resonator ground state and a qubit ${ }^{3}$ as well as permitting cooling and squeezing of the resonator modes and enabling quantum-limited position detection ${ }^{4-10}$. In spite of these intriguing possibilities, no experimental realization of an on-chip, coupled mechanical-resonator-SQUID system has yet been achieved. Here, we demonstrate sensitive detection of the position of a $2 \mathrm{MHz}$ flexural resonator that is embedded into the loop of a d.c. SQUID. We measure the resonator's thermal motion at millikelvin temperatures, achieving an amplifier-limited displacement sensitivity of $10 \mathrm{fm} \mathrm{Hz}^{-1 / 2}$ and a position resolution that is $\mathbf{3 6}$ times the quantum limit.

d.c. superconducting quantum interference devices (SQUIDs) consist of a superconducting loop with two Josephson junctions ${ }^{1}$. The voltage across the d.c. SQUID does not depend only on the current through it, but also on the magnetic flux piercing through the loop, enabling tiny changes in the magnetic field to be detected. Besides this more common application, the d.c. SQUID should also be able to detect small variations in the area of its loop due to the motion of an integrated flexural resonator in the presence of a static magnetic field. Our Nb-based d.c.-SQUID displacement detector is based on this principle and is shown in Fig. 1a,b and described in the Methods section. Its potential displacement sensitivity can be estimated as follows. The resonator has a length of $\ell=50 \mu \mathrm{m}$ and the loop is placed in a magnetic field of $B=0.1$ T oriented as described in Fig. 1d. A deflection $u=1 \mathrm{fm}$ of the resonator will then result in a change in flux through the loop of the order of $B \ell u=2.5 \mu \Phi_{0}$, where $\Phi_{0}=h / 2 e=2.07 \mathrm{fT} \mathrm{m}^{2}$ is the flux quantum. Low-temperature d.c. SQUIDs have a typical flux sensitivity of $10^{-6} \Phi_{0} \mathrm{~Hz}^{-1 / 2}$ (ref. 11), which is sufficient to reach a displacement sensitivity of $0.4 \mathrm{fm} \mathrm{Hz}^{-1 / 2}$. This places the d.c.-SQUID detector in the same league as other highly sensitive on-chip position detectors ${ }^{12-15}$.

Before using the d.c. SQUID as a displacement detector, its characteristics are determined to find a proper bias point. Figure 1d shows a schematic diagram of the measurement set-up, which is described in detail in the Methods section. A bias current $I_{\mathrm{B}}$ is applied to the d.c. SQUID and its output voltage $V$ is measured. The d.c. SQUID produces a non-zero output voltage once $I_{\mathrm{B}}$ exceeds the d.c.-SQUID's critical current $I_{\mathrm{C}}$, which depends on the magnetic flux bias $\Phi$ through the d.c.-SQUID loop and on the

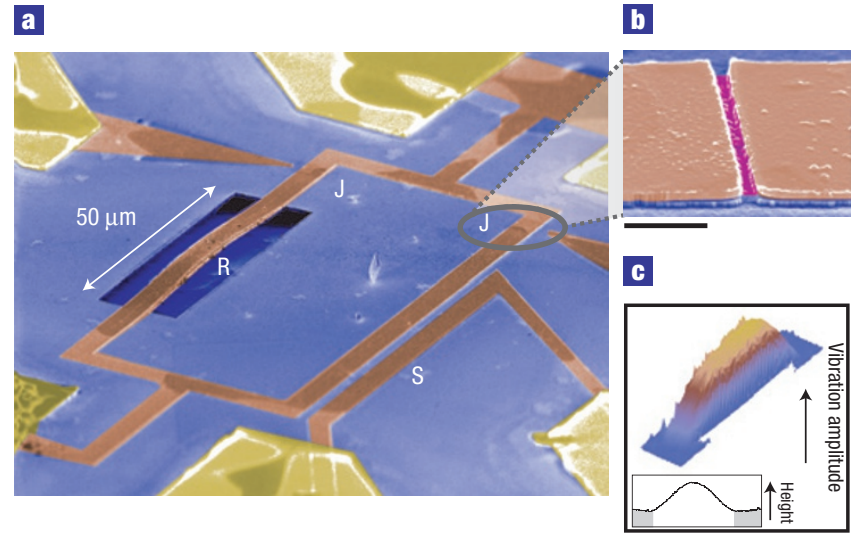

d

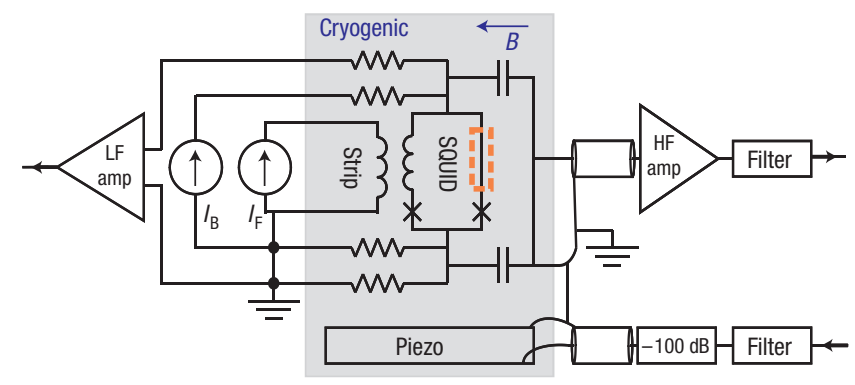

Figure 1 The coupled d.c.-SQUID-resonator geometry and measurement set-up. a, Colourized scanning electron micrograph of a device at $80^{\circ}$ inclination. The beam resonator $(\mathrm{R})$ is buckled away from the substrate owing to compressive strain (see the Supplementary Information). The stripline (S) is used to change the flux bias through the d.c. SQUID. The Nb-InAs weak links (J) are located adjacent to unused $\mathrm{Nb}$ side gates. $\mathbf{b}$, Colourized scanning electron micrograph of one of the 200-nm-long Nb-InAs-Nb junctions. The scale bar is $1 \mu \mathrm{m}$. c, A three-dimensional image of the vibration amplitude of the driven beam resonance at $2 \mathrm{MHz}$, acquired at room temperature using dynamic force microscopy. The inset shows the static buckling profile of the beam with a maximum deflection of $1.5 \mu \mathrm{m}$ measured using atomic force microscopy. $\mathbf{d}$, Schematic overview of the measurement circuit. A coupling field $B$ is applied parallel to the d.c.-SQUID plane and perpendicular to the length direction of the resonator (marked by the red dashed rectangle). The sample is glued onto a piezo actuator, which is connected to a filtered voltage source for the driven measurements. HF (LF) amp: high-frequency (low-frequency) amplifier. 

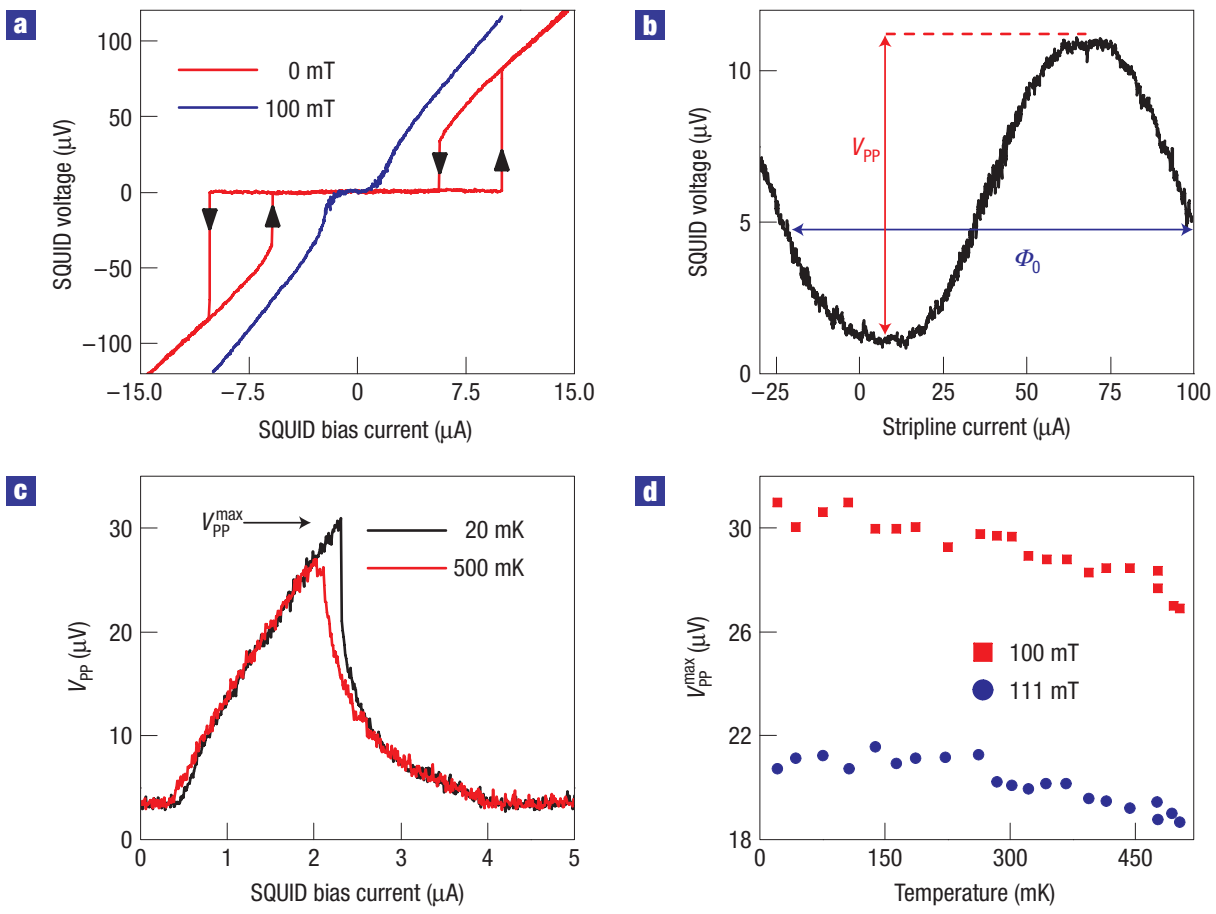

Figure 2 Characteristics of the d.c. SQUID. a, d.c.-SQUID voltage as a function of the bias current $I_{\mathrm{B}}$ at $20 \mathrm{mK}$. At zero magnetic field, the d.c. SQUID exhibits hysteretic behaviour (red curves), which indicates that the d.c. SQUID is underdamped. The blue curve shows the voltage-current response at $100 \mathrm{mT}$, which is the lowest of two fields used for position detection. The increased magnetic field has suppressed the hysteresis owing to a reduction of the critical current. b, Average d.c.-SQUID voltage as a function of the applied stripline current at $100 \mathrm{mT}$ and $I_{\mathrm{B}}=2.5 \mu \mathrm{A}$. The peak-to-peak voltage swing is $V_{\mathrm{Pp}}$. C, Measurements of $V_{\mathrm{Pp}}$ as a function of $I_{\mathrm{B}}$ at $B=100 \mathrm{mT}$. The parameters $I_{0}$ and $V_{\mathrm{PP}}^{\max }$ obtained from this measurement are used to tune the d.c. SQUID to a sensitive bias point. $\mathbf{d}, V_{\mathrm{PP}}^{\max }$ as a function of refrigerator temperature at two different magnetic fields.

critical current of the individual junctions, $I_{0}$. Figure 2 a shows that at $B=0 \mathrm{~T}$ the d.c.-SQUID's $V-I_{\mathrm{B}}$ relationship exhibits hysteresis, indicating that the d.c. SQUID is underdamped ${ }^{1}$. This hysteresis must be suppressed to operate the d.c. SQUID as a sensitive linear flux detector. This is achieved by increasing $B$, which decreases the critical current of the junctions ${ }^{16}$. We find that for $B \geq 0.1 \mathrm{~T}$ the d.c. SQUID is sufficiently damped such that no hysteresis is observed (Fig. 2a). Note that in this detection scheme the d.c. SQUID is biased above the critical current. It can in principle also be used as a position detector when it is biased below the critical current, where it acts as a tunable inductor ${ }^{4}$.

The d.c. SQUID is most sensitive to changes in the magnetic flux when it is tuned to a working point with a steep slope of $V(\Phi)$. Figure $2 \mathrm{~b}$ shows the relation between $V$ and the applied stripline current $I_{\mathrm{F}}$ that changes $\Phi$. For a d.c. SQUID, both $I_{\mathrm{C}}$ and $V$ depend periodically on $\Phi$ with a period of $\Phi_{0}$. The steepest slope occurs approximately half-way between the minimum and maximum of the voltage swing. The difference between these two voltages is the peak-to-peak voltage swing $V_{\mathrm{PP}}$. Figure $2 \mathrm{c}$ shows $V_{\mathrm{PP}}$ as a function of $I_{\mathrm{B}}$, where $V_{\mathrm{PP}}^{\max }$ is the maximum voltage swing. Numerical simulations (see the Supplementary Information) show that this maximum occurs at $I_{\mathrm{B}}=2 I_{0}$.

In our experiment there is a slow flux drift, which makes it difficult to maintain a constant flux bias by simply applying a constant stripline current. Therefore, a feedback loop is used that maintains a constant average setpoint voltage $\langle V\rangle=V_{\mathrm{SP}}$ by adjusting $I_{\mathrm{F}}$ for a given value of $I_{\mathrm{B}}$. An added advantage of using the feedback loop is that it compensates external low-frequency $(<1 \mathrm{kHz})$ flux noise.
Numerical analysis (see the Supplementary Information) reveals that the ratios $I_{\mathrm{B}} / I_{0}$ and $V_{\mathrm{SP}} / V_{\mathrm{PP}}^{\max }$, needed to achieve the maximum value of the flux responsivity $\mathrm{d} V / \mathrm{d} \Phi$, are approximately constant. This allows us to apply a bias that gives maximum $\mathrm{d} V / \mathrm{d} \Phi$ even if $I_{0}$ and $V_{\mathrm{PP}}^{\max }$ are changing owing to temperature variations as shown in Fig. $2 \mathrm{~d}$. In practice, values for $V_{\mathrm{SP}}$ and $I_{\mathrm{B}}$ must be chosen such that they allow stable operation of the feedback loop in addition to maximizing $\mathrm{d} V / \mathrm{d} \Phi$. The values $I_{\mathrm{B}}=2 I_{0}$ and $V_{\mathrm{SP}}=V_{\mathrm{PP}}^{\max } / 2$ are found to provide a good balance between these requirements.

As the resonator is integrated into the d.c. SQUID, the flux through the loop depends on the position $u$ of the fundamental out-of-plane mode of the beam according to $\Phi=\Phi_{a}+a B \ell u$, where $\Phi_{a}$ is the applied flux when the resonator is in its equilibrium position and $a$ is a geometrical factor that depends on the mode shape. The position $u$ is defined such that the effective spring constant of the mode is $k_{\mathrm{R}}=m\left(2 \pi f_{\mathrm{R}}\right)^{2}$, where $m$ is the total mass of the beam and $f_{\mathrm{R}}$ is the resonance frequency of the mode. Atomic force microscopy shows that the resonator is buckled (Fig. 1c, inset) and we use a continuum model to find $a=0.91$ (see the Supplementary Information). In our configuration, the d.c. SQUID functions as a linear displacement detector in the small-signal limit $a B \ell u \ll \Phi_{0}$ with a displacement responsivity $\mathrm{d} V / \mathrm{d} u=(\mathrm{d} V / \mathrm{d} \Phi)(\mathrm{d} \Phi / \mathrm{d} u)$.

The resonance frequency $f_{\mathrm{R}}$ is located by driving the resonator with a piezo actuator (Fig. 1d) and monitoring the resulting output voltage of the d.c. SQUID. The resonance frequency is $2.0018 \mathrm{MHz}$ with a Lorentzian amplitude response (Fig. 3a). Room-temperature dynamic force microscopy ${ }^{17}$ confirms that this is indeed the 

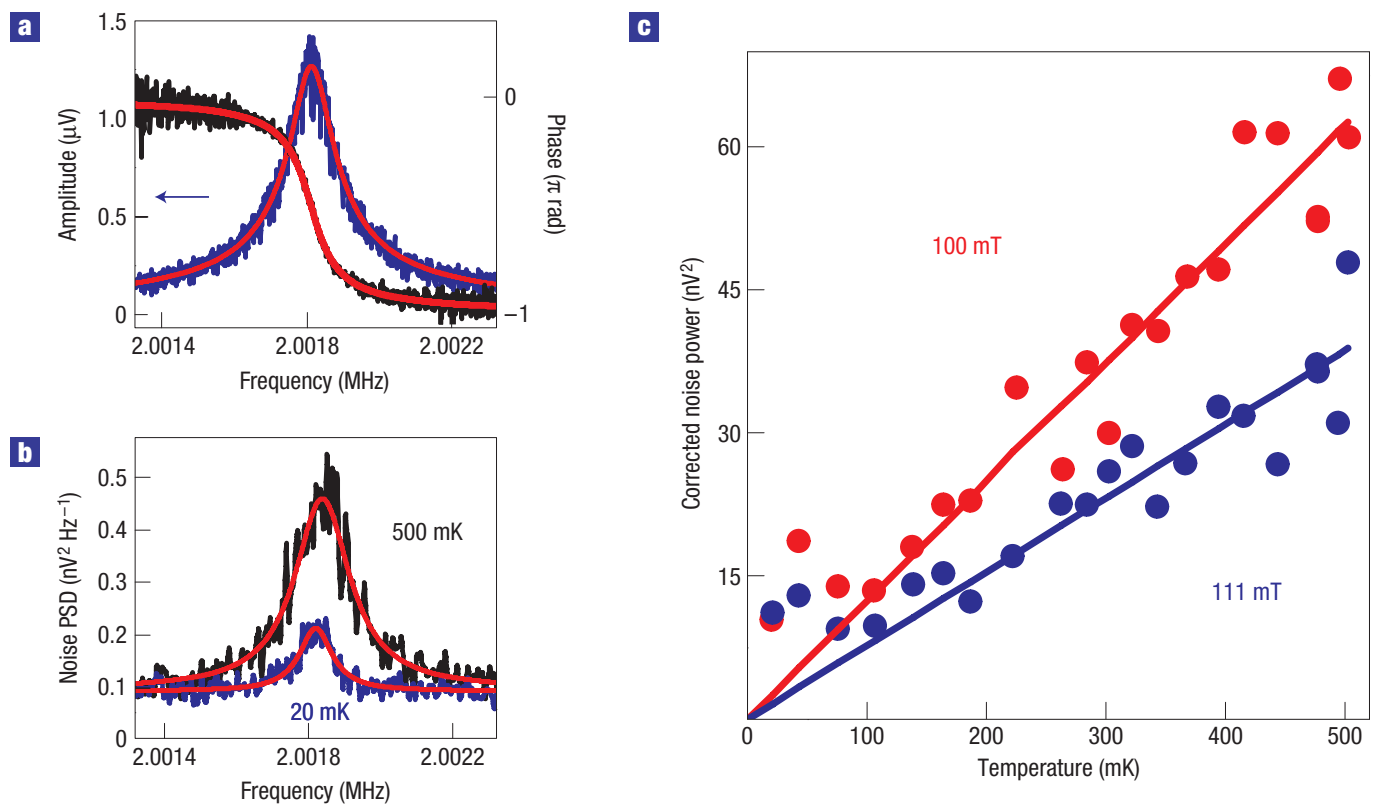

Figure 3 Driven response and Brownian motion of the resonator. a, The driven resonator response at $T=20 \mathrm{mK}$ and $B=100 \mathrm{mT}$. Amplitude and phase data are shown in blue and black, respectively. The amplitude and phase response are well fitted by the response of a harmonic oscillator (red). The peak amplitude corresponds to approximately $20 \mathrm{pm}$ deflection. b, Noise power spectral density around the beam resonance frequency at $100 \mathrm{mT}$ for two different temperatures. The peak in the noise spectra is due to the Brownian motion of the resonator. c, The corrected voltage noise power $\left\langle V_{\mathrm{R}}^{\prime 2}\right\rangle$ extracted from the thermal spectra at $100 \mathrm{mT}$ (red) and $111 \mathrm{mT}$ (blue). The solid lines indicate a linear least-mean-squares fit to the noise powers for temperatures above $100 \mathrm{mK}$. The linear relationship is predicted by the equipartition theorem and its slope is used to calibrate the deflection responsivity of the flux-based transducer. The steeper line for the $100 \mathrm{mT}$ data indicates a higher responsivity than at $111 \mathrm{mT}$. The lowest achieved resonator temperature is $84 \mathrm{mK}$ at a refrigerator temperature of $20 \mathrm{mK}$. This difference implies that the resonator does not thermalize at the lowest temperatures (see the Supplementary Information).

fundamental mode (Fig. 1c) and it is also in good agreement with our continuum model. From a least-squares Lorentzian fit, we extract a quality factor $Q=1.8 \times 10^{4}$ at $B=100 \mathrm{mT}$ and refrigerator temperature $T=20 \mathrm{mK}$. For all measurements, the peak voltage amplitude is much smaller than $V_{\mathrm{PP}}^{\max }$ (Fig. 2d), which means that the flux oscillations due to the resonator are within the small-signal limit.

Thermomechanical noise thermometry is used to calibrate the deflection responsivity ${ }^{13-15}$. Without actively driving the resonator, the noise power spectral density of the output voltage is acquired around the mechanical resonance frequency. The spectra in Fig. 3b show a constant background on which a Lorentzian peak is superimposed. This peak is caused by the Brownian motion of the beam. The quality factor and resonance frequency are identical to those of the driven response (Fig. 3a). The voltage noise power due to the resonator $\left\langle V_{\mathrm{R}}^{2}\right\rangle$, the area underneath the peak, is extracted from the fitted response function.

The noise spectrum is measured in the temperature range $20 \mathrm{mK}<T<500 \mathrm{mK}$. To compare the noise power at different temperatures, $\left\langle V_{\mathrm{R}}^{2}\right\rangle$ must be corrected for differences in $\mathrm{d} V / \mathrm{d} \Phi$. The flux responsivity is proportional to $V_{\mathrm{PP}}^{\max }$ and thus has the same temperature dependence (Fig. 2d). This enables the introduction of the corrected voltage noise power $\left\langle V_{\mathrm{R}}^{\prime 2}\right\rangle=\left\langle V_{\mathrm{R}}^{2}\right\rangle \cdot\left(V_{\mathrm{pP}}^{\max }(20 \mathrm{mK}) / V_{\mathrm{PP}}^{\max }(T)\right)^{2}$. The corrected noise powers in Fig. 3c show a linear decrease with temperature down to $100 \mathrm{mK}$. The slope $S(B)$ from a linear fit to this data, combined with the equipartition theorem gives the deflection responsivity $\mathrm{d} V / \mathrm{d} u=V_{\mathrm{PP}}^{\max }(T) / V_{\mathrm{PP}}^{\max }(20 \mathrm{mK}) \cdot\left(S(B) k_{\mathrm{R}} / k_{\mathrm{B}}\right)^{1 / 2}$, where $k_{\mathrm{B}}$ is the Boltzmann constant and $k_{\mathrm{R}}=97 \mathrm{~N} \mathrm{~m}^{-1}$, using $m=6.1 \times 10^{-13} \mathrm{~kg}$. The resulting displacement responsivities at $20 \mathrm{mK}$ are $3.0 \times 10^{-2} \mathrm{nV} \mathrm{fm}^{-1}$ and $2.3 \times 10^{-2} \mathrm{nV} \mathrm{fm}^{-1}$ for $B=100 \mathrm{mT}$ and $111 \mathrm{mT}$ respectively. The fact that the displacement responsivity at $111 \mathrm{mT}$ is lower than at $100 \mathrm{mT}$ might seem counter-intuitive, but the larger flux change for the same displacement is compensated by a stronger reduction of $V_{\mathrm{PP}}^{\max }$, as shown in Fig. 2d. The best displacement sensitivity coincides with the highest responsivity because the flux-based position detector is limited by the noise floor of the room-temperature voltage amplifier $\sqrt{S_{\mathrm{V}}}$. The highest observed sensitivity is $\sqrt{S_{u}}=(\mathrm{d} V / \mathrm{d} u)^{-1} \sqrt{S_{\mathrm{V}}}=10 \mathrm{fm} \mathrm{Hz}^{-1 / 2}$ and occurs at $20 \mathrm{mK}$ and $100 \mathrm{mT}$.

It is interesting to calculate the position resolution of the detector and compare it with the fundamental limit imposed by quantum mechanics ${ }^{18}$. The position resolution is $\Delta u=\sqrt{S_{u} \Delta f}$, where $\Delta f=\pi f_{\mathrm{R}} / 2 Q$ is the effective noise bandwidth of the resonator. This yields $\Delta u=133 \mathrm{fm}$ for the highest observed sensitivity of the detector. The quantum limit for the position resolution of a continuous linear detector is $\Delta u_{\mathrm{QL}}=\sqrt{\hbar / m\left(2 \pi f_{\mathrm{R}}\right)}=4 \mathrm{fm}$ for our resonator ${ }^{19}$, so that the detector resolution is a factor $\Delta u / \Delta u_{\mathrm{QL}}=36$ from the quantum limit. The resonator enters the quantum regime once it has a thermal occupation factor $N \approx T_{\mathrm{R}} / T_{\mathrm{Q}}<1$, where $T_{\mathrm{Q}}=h f_{\mathrm{R}} / k_{\mathrm{B}}$ is the quantum temperature and $T_{\mathrm{R}}$ is the resonator temperature, which can be found from the voltage noise power using $T_{\mathrm{R}}=\left\langle V_{\mathrm{R}}^{2}\right\rangle k_{\mathrm{R}} / k_{\mathrm{B}}\langle\mathrm{d} V / \mathrm{d} u\rangle^{2}$. From the data in Fig. $3 \mathrm{c}$, the lowest observed resonator temperature is $T_{\mathrm{R}}=84 \mathrm{mK}$, which yields $N=878$.

There are two major challenges for observing quantum behaviour in a macroscopic mechanical resonator ${ }^{20}$ a quantum-limited position detector ${ }^{18,19,21}$ with resolution 
$\Delta u=\Delta u_{\mathrm{QL}}$ and a resonator with $N<1$. These requirements can be simultaneously met by our device configuration. The quantum mechanical ground state for a $1 \mathrm{GHz}$ resonator is reached at temperatures below $T_{\mathrm{Q}}=45 \mathrm{mK}$, which can be achieved in a dilution refrigerator. The d.c. SQUID is known to be a near quantum-limited flux detector and flux sensitivities of $\sqrt{S_{\Phi}}=0.01 \mu \Phi_{0} \mathrm{~Hz}^{-1 / 2}$ are possible ${ }^{22}$. For the current device $\sqrt{S_{\Phi}} \sim 10 \mu \Phi_{0} \mathrm{~Hz}^{-1 / 2}$ is limited by the room- temperature amplifier. Thus, by reducing the amplifier noise floor by using, for example, a second d.c. SQUID as an amplifier and by increasing the flux responsivity of the first d.c. SQUID, the sensitivity may ultimately be improved by three orders of magnitude. Under these conditions, a $1 \mathrm{GHz}$ resonator made of a 300-nm-long InAs beam with $Q=1,000$ will require a magnetic field of $1 \mathrm{~T}$ for the detector to reach quantum-limited sensitivity. Note that InAs is very suitable for such a resonator, as beams that are only tens of nanometres wide can still carry a substantial supercurrent ${ }^{23}$. d.c.-SQUID operation in the required high magnetic field may be possible by using narrow and thin $\mathrm{Nb}$ lines ${ }^{24}$. With these improvements, our flux-based measurement method can potentially be extended to detect the resonator's ground state.

\section{METHODS}

\section{DEVICE DETAILS}

The Nb of the d.c.-SQUID loop and the stripline is evaporated (thickness $100 \mathrm{~nm}$ ) onto a thin InAs-AlGaSb heterostructure that has been grown epitaxially on a GaAs(111)A substrate (see the Supplementary Information and ref. 25). The inner area of the loop is $40 \mu \mathrm{m} \times 80 \mu \mathrm{m}$ and the line width is $4 \mu \mathrm{m}$. The stripline is placed $1.5 \mu \mathrm{m}$ from the d.c. SQUID and runs $70 \mu \mathrm{m}$ parallel to the d.c.-SQUID loop. Weak links are formed at two points where the $\mathrm{Nb}$ is interrupted (Fig. 1b). At these points, the current flows through the InAs surface layer, thus forming an SNS-type Josephson junction ${ }^{26}$. A dry-etch is used to remove the conducting InAs layer everywhere except underneath the metallized parts and at the junctions. Electrical contact to the $\mathrm{Nb}$ is made by evaporation of $20 \mathrm{~nm} \mathrm{Ti}$ and $200 \mathrm{~nm}$ Au. The flexural beam resonator is made by removing the GaAs substrate underneath part of the loop with a wet-etch. The resulting beam is buckled away from the substrate owing to compressive strain (Fig. 1c).

\section{MEASUREMENT SET-UP}

The device is mounted in a dilution refrigerator and the coarse magnetic field $B$ is applied using a superconducting solenoid magnet. The low-frequency circuit is used to set the d.c. SQUID to a working point by applying a bias current $I_{\mathrm{B}}$ through the d.c. SQUID and a current $I_{\mathrm{F}}$ through the stripline. The battery-powered current sources and low-frequency voltage amplifier are optically isolated from mains-operated equipment. The high-frequency d.c.-SQUID output voltage at the resonator frequency is measured using a two-stage room-temperature amplifier with $80 \mathrm{~dB}$ gain, $10 \mathrm{k} \Omega$ input impedance and equivalent input voltage noise $\sqrt{S_{\mathrm{V}}}=0.3 \mathrm{nV} \mathrm{Hz}^{-1 / 2}$. The high-frequency and low-frequency circuits are separated by $1 \mathrm{k} \Omega$ resistors and $10 \mathrm{nF}$ capacitors at the cryogenic stage (Fig. 1d).
Received 3 June 2008; accepted 28 July 2008; published 31 August 2008.

References

1. Clarke, J. \& Braginski, A. I. The SQUID Handbook Vol. 1 (Wiley-VCH, GmbH and Co. KGaA Weinheim, 2004).

2. Makhlin, Y., Schon, G. \& Shnirman, A. Quantum-state engineering with Josephson-junction devices. Rev. Mod. Phys. 73, 357-400 (2001).

3. Xue, F. et al. Controllable coupling between flux qubit and nanomechanical resonator by magnetic field. New J. Phys. 9, 35 (2007).

4. Blencowe, M. P. \& Buks, E. Quantum analysis of a linear DC SQUID mechanical displacement detector. Phys. Rev. B 76, 014511 (2007).

5. Zhou, X. \& Mizel, A. Nonlinear coupling of nanomechanical resonators to Josephson quantum circuits. Phys. Rev. Lett. 97, 267201 (2006).

6. Buks, E., Segev, E., Zaitsev, S., Abdo, B. \& Blencowe, M. Quantum nondemolition measurement of discrete Fock states of a nanomechanical resonator. Europhys. Lett. 81, 10001 (2007).

7. Blencowe, M. P. \& Buks, E. Decoherence and recoherence in a vibrating RF SQUID. Phys. Rev. B 74, 174504 (2006).

8. Wang, Y., Semba, K. \& Yamaguchi, H. Cooling of a micro-mechanical resonator by the back-action of Lorentz force. New J. Phys. 10, 043015 (2008).

9. Huo, W. Y. \& Long, G. L. Generation of squeezed states of nanomechanical resonator using three-wave mixing. Appl. Phys. Lett. 92, 133102 (2008).

10. Xue, F., Liu, Y., Sun, C. P. \& Nori, F. Two-mode squeezed states and entangled states of two mechanical resonators. Phys. Rev. B 76, 064305 (2007).

11. Mück, M., Kycia, J. B. \& Clarke, J. Superconducting quantum interference device as a near-quantum-limited amplifier at $0.5 \mathrm{GHz}$. Appl. Phys. Lett. 78, 967-969 (2001).

12. Knobel, R. G. \& Cleland, A. N. Nanometre-scale displacement sensing using a single electron transistor. Nature 424, 291-293 (2003).

13. LaHaye, M. D., Buu, O., Camarota, B. \& Schwab, K. C. Approaching the quantum limit of a nanomechanical resonator. Science 304, 74-77 (2004).

14. Flowers-Jacobs, N. E., Schmidt, D. R. \& Lehnert, K. W. Intrinsic noise properties of atomic point contact displacement detectors. Phys. Rev. Lett. 98, 096804 (2007).

15. Regal, C. A., Teufel, J. D. \& Lehnert, K. W. Measuring nanomechanical motion with a microwave cavity interferometer. Nature Phys. 4, 555-560 (2008).

16. Cuevas, J. C. \& Bergeret, F. S. Magnetic interference patterns and vortices in diffusive SNS junctions. Phys. Rev. Lett. 99, 217002 (2007).

17. Garcia-Sanchez, D. et al. Mechanical detection of carbon nanotube resonator vibrations. Phys. Rev. Lett. 99, 085501 (2007).

18. Caves, C. M., Thorne, K. S., Drever, W. P., Sandberg, V. D. \& Zimmermann, N. On the measurement of a weak classical force coupled to a quantum-mechanical oscillator. I. Issues of principle. Rev. Mod. Phys. 52, 341-392 (1980).

19. Caves, C. M. Quantum limits on noise in linear amplifiers. Phys. Rev. D 26, 1817-1839 (1981).

20. Schwab, K. C. \& Roukes, M. L. Putting mechanics into quantum mechanics. Phys. Today 58, 36-42 (2005).

21. Clerk, A. A. Quantum-limited position detection and amplification: A linear response perspective. Phys. Rev. B 70, 245306 (2004).

22. Koch, R. H., van Harlingen, D. J. \& Clarke, J. Quantum noise theory for the d.c.-SQUID. Appl. Phys. Lett. 38, 380-382 (1980).

23. Doh, Y. et al. Tunable supercurrent through semiconductor nanowires. Science 309, 272-275 (2005).

24. Stan, G., Field, S. B. \& Martinis, J. M. Critical field for complete vortex expulsion from narrow superconducting strips. Phys. Rev. Lett. 92, 097003 (2004).

25. Yamaguchi, H., Miyashita, S. \& Hirayama, Y. Microelectromechanical displacement sensing using InAs-AlGaSb heterostructures. Appl. Phys. Lett. 82, 394-396 (2003).

26. Takayanagi, H. \& Kawakami, T. Superconducting proximity effect in the native inversion layer on InAs. Phys. Rev. Lett. 54, 2449-2452 (1985).

Supplementary Information accompanies the paper at www.nature.com/naturephysics.

\section{Acknowledgements}

We thank Y. Blanter for discussions, T. Akazaki, H. Okamoto and K. Yamazaki for help with fabrication, R. Schouten for the measurement electronics and A. Bachtold and D. Sanchez-Garcia for advice on the dynamic force microscopy measurement. This work was supported in part by JSPS KAKENHI (20246064 and 18201018), FOM, a Dutch NWO VICI grant and NanoNed.

\section{Author information}

Reprints and permission information is available online at http://npg.nature.com/reprintsandpermissions. Correspondence and requests for materials should be addressed to S.E. or H.S.J.v.d.Z. 